\title{
Exploiting Hopping Pilots for Parametric Channel Estimation in OFDM Systems
}

\author{
M. R. Raghavendra, S. Bhashyam, and K. Giridhar
}

\begin{abstract}
In this letter, we investigate the effect of hopping pilots on the parametric channel estimation in orthogonal frequency-division multiplexing (OFDM) systems. Channel estimation algorithms based on parametric channel modeling require the multipath delays to be estimated at the receiver. The use of a fixed pilot pattern leads to longer training overhead in multipath delay estimation for slow fading channels. However, if hopping pilot patterns are available, then we show that the normalized mean-squared error (NMSE) convergence rate of the channel estimates can be significantly improved. We also show that hopping pilot patterns in OFDM systems effectively allow the eigenvectors of the delay subspace (of the autocorrelation matrix) to be estimated faster. Simulation results are provided to show the faster convergence rate of the NMSE for the hopping pilot pattern over the fixed pilot pattern.
\end{abstract}

Index Terms-Fading multipath channels, hopping pilots, orthogonal frequency-division multiplexing (OFDM), parametric estimation, subspace channel estimation.

\section{INTRODUCTION}

$\mathbf{O}$ RTHOGONAL frequency division multiplexing (OFDM) has received considerable attention for its advantages in high bit-rate transmission over frequency-selective channels. The presence of a cyclic prefix (CP) completely eliminates intersymbol interference and replaces the complex time-domain equalizer by a simple single tap frequency-domain equalizer.

Channel estimation techniques based on the sample spaced channel models (where multipath delays are integer multiples of sampling period) have been proposed in [2]. A least-squares (LS) technique for sparse channels is proposed in [3]. However, these methods suffer from irreducible interpolation errors when the multipath delays are nonsample spaced [1]. Channel estimation based on parametric channel modeling is proposed in [4] using minimum description length to estimate the number of paths and estimation of signal parameters using rotational invariance technique (ESPRIT) to estimate the initial multipath delays. This method removes the error floor due to discrete Fourier transform (DFT)-based interpolation. However, in relatively slow fading channels, the number of OFDM symbols required for the multipath delay estimation will be large. Another parametric model-based method is proposed in [6].

Manuscript received February 18, 2005; revised June 10, 2005. This work was supported by a National Research Fellowship Grant from IETE, India, on Emerging Wireless Technologies. The associate editor coordinating the review of this manuscript and approving it for publication was Dr. Gerald Matz.

The authors are with the Telecommunications and Computer Networks (TeNeT) Group, Department of Electrical Engineering, Indian Institute of Technology Madras, Chennai 600 036, India (e-mail: raghumr@tenet.res.in; srikrishna@tenet.res.in; giri@ tenet.res.in).

Digital Object Identifier 10.1109/LSP.2005.856889
However, this method needs an exhaustive search to estimate the multipath delays and is rather impractical. A delay subspace tracking algorithm applicable for OFDM systems has been proposed in [5]. Unlike parametric estimation methods, this method does not suffer from impairments of nonlinear estimation, such as threshold effects and sensitivity to modeling errors. However, it suffers from error floor due to DFT-based interpolation. We have addressed the problem of slow convergence in [4] and error floor due to DFT-based channel interpolation in [5].

Pilot hopping has been adopted in IEEE 802.16 and Digital Video Broadcasting (DVB) standards [12]. In these standards, hopping pilots are used to enhance time and frequency synchronization and also to meet the Nyquist criterion for channel sampling in frequency domain. In this letter, we exploit pilot hopping in parametric channel estimation. The key advantages of using pilot hopping in parametric channel estimation are as follows. 1) The number of OFDM symbols required to estimate the multipath components is significantly lower than [4]. 2) For a multipath channel with $L$ independent paths, only $L$ pilot subcarriers are required to ensure proper channel estimation. It must be noted that existing parametric channel estimators for OFDM (e.g., [4]) usually require more than $L$ pilot subcarriers per symbol.

\section{A. Basic Notation}

Boldface letters denote vectors or matrices; $(\cdot)^{T},(\cdot)^{*},(\cdot)^{H}$, and $(\cdot)^{\dagger}$ denote transpose, complex conjugate, Hermitian, and pseudo-inverse, respectively; $\|\cdot\|$ denotes the Euclidean norm; $E[\cdot]$ denotes the expectation operator; $\mathbf{I}_{K}$ denotes the $K \times K$ identity matrix; $\mathbf{0}_{P}$ denotes the column vector of size $P$ with zero entries, and $\operatorname{diag}(\mathbf{x})$ is the diagonal matrix with elements of the vector $\mathbf{x}$ on its main diagonal.

\section{Channel Estimation}

We consider an OFDM system with $K$ subcarriers over a bandwidth of $B \mathrm{~Hz}$, where the received signal is sampled every $T=(1 / B)$ seconds.

The wireless channel is modeled to have $L$ multipath components, where each path is characterized by a gain factor $h_{l}$ and a delay $\tau_{l}$ [4], and has the form $h(\tau)=\sum_{l=0}^{L-1} h_{l} \delta\left(\tau-\tau_{l}\right)$. The path gains $\left\{h_{l}\right\}_{l=0}^{l=L-1}$ are zero-mean complex Gaussian random variables with $E\left[h_{l} h_{m}^{*}\right]=0$ for $l \neq m$. The channel is assumed to be quasi-static (multipath gain remains constant over a symbol and could vary symbol to symbol), and multipath delays are considered as constants for a large number of OFDM symbols [5, Sec. III-A]. The channel delay spread is assumed 
to be smaller than the $\mathrm{CP}$ of the OFDM symbol $\left(L \leq L_{\mathrm{cp}}\right)$. In frequency domain, the channel is described by the Fourier transform $H[k]$ of $h(\tau)$

$$
H[k]=\sum_{l=0}^{L-1} h_{l} e^{\frac{-j 2 \pi k \tau_{l}}{K T}} \quad \text { for } k=0,1, \ldots, K-1 .
$$

In matrix form, we can write the system of equations in (1) as $\mathbf{H}=\mathbf{F h}$, where $\mathbf{h}=\left(h_{0}, h_{1}, \ldots, h_{L-1}\right)^{T}$, and the $(l, k)$ th element of $\mathbf{F}$ is

$[\mathbf{F}]_{l, k}=e^{\frac{-j 2 \pi k \tau_{l}}{K T}} \quad$ for $\quad k=0,1, \ldots, K-1, l=0,1, \ldots, L-1$.

At the receiver, we write the $n$th measurement vector in frequency domain as

$$
\mathbf{Y}_{n}=\mathbf{X}_{n} \mathbf{H}_{n}+\mathbf{N}_{n}
$$

where $\mathbf{X}_{n}=\operatorname{diag}\left(X_{0, n}, X_{1, n}, \ldots, X_{K-1, n}\right), X_{i, n}$ denotes the data symbol on the $i$ th subcarrier of the $n$th OFDM symbol, and $\mathbf{H}_{n}=\mathbf{F} \mathbf{h}_{n}$ is the channel experienced by the $n$th OFDM symbol. The zero-mean complex Gaussian noise vector $\mathbf{N}_{n}$ has distribution $\mathbf{N}_{n} \sim \mathcal{C N}\left(\mathbf{0}, \sigma^{2} \mathbf{I}_{K}\right)$.

Let $K_{P}$ be the number of pilot subcarriers used in an OFDM symbol. In our discussion, we choose $K_{P}$ such that $P=K / K_{P}$ is an integer. For a fixed number of pilot subcarriers in an OFDM symbol, it has been shown that the mean-square error in the channel estimation is minimum if the pilots are equi-spaced and equi-powered on the frequency grid [7]. The subcarrier indices for the $i$ th pilot pattern are

$$
\mathcal{I}_{i}=\{i+j P\} \quad \text { for } j \in\left[0, K_{P}-1\right] \quad \text { and } \quad i \in[0, P-1] \text {. }
$$

Considering only pilot subcarrier positions in (2), we have

$$
\mathbf{Y}_{n}^{\prime}=\mathbf{X}_{n}^{\prime} \mathbf{H}_{n}^{\prime}+\mathbf{N}_{n}^{\prime}
$$

where $\mathbf{H}_{n}^{\prime}=\mathbf{F}_{n}^{\prime} \mathbf{h}_{n}$, and $\mathbf{F}_{n}^{\prime}$ is obtained by retaining the rows of $\mathbf{F}$ corresponding to the pilot positions of the $n$th OFDM symbol.

The LS channel estimates of $\mathbf{H}_{n}^{\prime}$ are given by [1]

$$
\hat{\mathbf{H}}_{l s, n}^{\prime}=\left(\mathbf{X}_{n}^{\prime}\right)^{-1} \mathbf{Y}_{n}^{\prime}=\mathbf{H}_{n}^{\prime}+\mathbf{W}_{n}^{\prime}
$$

where $\mathbf{W}_{n}^{\prime}=\left(\mathbf{X}_{n}^{\prime}\right)^{-1} \mathbf{N}_{n}$ is distributed as $\mathbf{W}_{n}^{\prime} \sim$ $\mathcal{C N}\left(\mathbf{0},\left(\sigma^{2} / \Omega\right) \mathbf{I}_{K_{P}}\right)$, and $\Omega$ is the power on each pilot subcarrier. Having obtained the channel estimates on the pilot subcarriers, we have to interpolate this over the entire frequency grid in order to define the equalizer for the data. The error floor introduced by the DFT-based interpolation methods [5] can be removed by using channel estimation based on the parametric channel model. The parametric channel estimator needs the multipath delays to be estimated at the receiver. The multipath delays can be estimated from the frequency-domain autocorrelation matrix $\mathbf{R}$ of the channel estimates $\hat{\mathbf{H}}_{l s, n}^{\prime}$.

\section{A. Estimation of Autocorrelation Matrix $\mathbf{R}$}

The sample autocorrelation matrix $\mathbf{R}$ is estimated using ergodic assumptions as

$$
\hat{\mathbf{R}}=\frac{1}{N} \sum_{n=1}^{N} \hat{\mathbf{H}}_{l s, n}^{\prime} \hat{\mathbf{H}}_{l s, n}^{\prime H}
$$

where $N$ is the number of OFDM symbols considered for averaging. In (6), if the pilot pattern is fixed to one of the possible $P$ patterns in (3), we have

$$
\hat{\mathbf{R}}=\mathbf{F}_{1}^{\prime}\left(\frac{1}{N} \sum_{n=1}^{N} \mathbf{h}_{n} \mathbf{h}_{n}^{H}\right) \mathbf{F}_{1}^{\prime H}+\hat{\mathbf{R}}_{w}
$$

where $\hat{\mathbf{R}}_{w}=(1 / N) \sum_{n=1}^{N}\left(\mathbf{W}_{n}^{\prime} \mathbf{W}_{n}^{\prime H}+\mathbf{H}_{n}^{\prime} \mathbf{W}_{n}^{\prime H}+\mathbf{W}_{n}^{\prime} \mathbf{H}_{n}^{\prime H}\right)$. $\mathbf{R}_{w}=\lim _{N \rightarrow \infty} \hat{\mathbf{R}}_{w}$ and has limiting distribution given by $\mathbf{R}_{w} \sim \mathcal{C N}\left(\mathbf{0},\left(\sigma^{2} / \Omega\right) \mathbf{I}_{K_{p}}\right)$. Observe that $\hat{\mathbf{R}}$ will always be full rank because of the noise term in (7). However, we are interested only in ensuring that the dominant eigenvalues of $\hat{\mathbf{R}}$ reveal the correct rank corresponding to all the independent multipath components. In case of slow fading channels, $\hat{\mathbf{R}}$ reveals correct rank only with averaging over a large number of OFDM symbols.

1) Pilot Hopping for Fast Estimation of $\mathbf{R}$ : The number of OFDM symbols required for the efficient estimation of $\mathbf{R}$ can be reduced by simulating the effect of fading at the receiver. This is done by hopping pilot subcarriers across successive OFDM symbols at the transmitter. The pilots are hopped in such a way that the subspace of the vector $\mathbf{H}_{n}^{\prime}$ is preserved for all $n$. To illustrate this, consider the case where the pilot positions are hopped by one subcarrier position (to the right) of every OFDM symbol. From (6), we have

$$
\hat{\mathbf{R}}=\frac{1}{N} \sum_{n=1}^{N} \mathbf{F}_{n}^{\prime} \mathbf{h}_{n} \mathbf{h}_{n}^{H} \mathbf{F}_{n}^{\prime H}+\hat{\mathbf{R}}_{w} .
$$

When the pilots are hopped by a subcarrier position, every OFDM symbol $\mathbf{F}_{n}^{\prime}$ and $\mathbf{F}_{n-1}^{\prime}$ are related by

$$
\mathbf{F}_{n}^{\prime}=\mathbf{F}_{n-1}^{\prime} \mathbf{D}
$$

where $\mathbf{D}=\operatorname{diag}\left(e^{\left(-j 2 \pi \tau_{0} / K T\right)}, \ldots, e^{\left(-j 2 \pi \tau_{L-1} / K T\right)}\right)$. Hence, we have

$$
\hat{\mathbf{R}}=\mathbf{F}_{1}^{\prime}\left(\frac{1}{N} \sum_{n=1}^{N} \mathbf{D}^{(n-1)} \mathbf{h}_{n} \mathbf{h}_{n}^{H}\left(\mathbf{D}^{H}\right)^{(n-1)}\right) \mathbf{F}_{1}^{\prime H}+\hat{\mathbf{R}}_{w}
$$

and $\mathbf{D}^{q}=\mathbf{D}^{q(\bmod P)}$ for $q=1, \ldots, N$.

Hopping pilot subcarriers from symbol to symbol emulates channel fading at the receiver by inducing phase rotation. This reduces the number of OFDM symbols used in the averaging of (6) for the efficient estimation of delay subspace basis. The phase rotation induced in the $l$ th path gain of $\mathbf{h}_{n}$ is $\tilde{h}_{l, n}=e^{\left(-j 2 \pi \tau_{l} / K T\right)} h_{l, n}$. Hence, for multipath channels with large delay spread, pilot hopping simulates more channel fading at the receiver, and hence, $\hat{\mathbf{R}}$ converges to $\mathbf{R}$ at a faster rate. This is shown with simulation in Fig. 3. 
2) Improving the $\hat{\mathbf{R}}$ : The forward/backward averaging given in [8] exploits the centrosymmetry property of the signal subspace of $\mathbf{R}$. This is given by $\hat{\mathbf{R}}^{f b}=(1 / 2)\left(\hat{\mathbf{R}}+\mathbf{J}(\hat{\mathbf{R}})^{*} \mathbf{J}\right)$, where $\mathbf{J}$ has 1's on antidiagonal elements and 0's elsewhere. The spatial smoothing technique [8] decorrelates the correlated narrowband or sinusoidal signals. In our current measurement model, spatial smoothing essentially provides a noise averaging and reduces the condition number of $\hat{\mathbf{R}}$. However, this smoothing requirement forces the number of pilot subcarriers to be more than $L$. Therefore, we use only the forward/backward averaging technique and no spatial smoothing.

\section{B. Estimating the Number of Paths and Delays}

In order to estimate the number of paths and delay subspace basis, an eigenvalue decomposition is performed on the estimated autocorrelation matrix as $\hat{\mathbf{R}}^{f b}=\sum_{m=1}^{K_{P}} \hat{\lambda}_{m} \hat{\mathbf{u}}_{m} \hat{\mathbf{u}}_{m}^{H}$, where $\hat{\lambda}_{1} \geq \hat{\lambda}_{2} \geq \cdots \geq \hat{\lambda}_{K_{P}}$ are the eigenvalues, and $\hat{\mathbf{u}}_{1}, \hat{\mathbf{u}}_{2}, \ldots, \hat{\mathbf{u}}_{K_{P}}$ are corresponding eigenvectors. The number of paths is estimated as the number of dominant eigenvalues of $\hat{\mathbf{R}}^{f b}$. The estimated eigenvalues are compared with noise variance $\left(\sigma^{2}\right)$, and those eigenvalues that exceed the noise variance by a factor of $\gamma$ are counted as dominant. Let $\hat{L}$ be the number of dominant eigenvalues of $\hat{\mathbf{R}}^{f b}$. The eigenvectors corresponding to $\hat{L}$ dominant eigenvalues forms the delay subspace basis $\hat{\mathbf{U}}_{s}=\left[\begin{array}{llll}\hat{\mathbf{u}}_{1} & \hat{\mathbf{u}}_{2} & \cdots & \hat{\mathbf{u}}_{\hat{L}}\end{array}\right]$.

The multipath delays are estimated by the ESPRIT algorithm [9] using the delay subspace basis vectors obtained from (12). The shift invariant subspaces are obtained from delay subspace as $\hat{\mathbf{U}}_{1}=\left[\begin{array}{ll}\mathbf{I}_{K_{P}-1} & \mathbf{0}_{K_{P}-1}\end{array}\right] \hat{\mathbf{U}}_{s}$ and $\hat{\mathbf{U}}_{2}=\left[\begin{array}{ll}\mathbf{0}_{K_{P}-1} & \mathbf{I}_{K_{P}-1}\end{array}\right] \hat{\mathbf{U}}_{s}$. The multipath delays are estimated as

$$
\hat{\tau}_{l}=\arg \left(\kappa_{l}^{*}\right) K T /(2 \pi P), \quad l=0, \ldots, \hat{L}-1
$$

where $\arg \left(\kappa_{l}^{*}\right)$ denotes the phase angle (in the range $[0,2 \pi)$ ) of $\kappa_{l}^{*}$, and $\left\{\kappa_{l}\right\}_{l=0}^{l=\hat{L}-1}$ are the eigenvalues of the matrix $\psi=$ $\left(\hat{\mathbf{U}}_{1}^{H} \hat{\mathbf{U}}_{1}\right)^{-1} \hat{\mathbf{U}}_{1}^{H} \mathbf{\mathbf { U }}_{2}$.

The estimated multipath delays $\left\{\hat{\tau}_{l}\right\}_{l=0}^{\hat{L}-1}$ are used in channel interpolation. The multipath delays are uniquely identified if $K_{P}>L$ and if $\tau_{L-1}<T K_{P}$ to prevent aliasing.

\section{Channel Interpolation}

The channel interpolation is done by frequency-domain filtering on the channel estimates $\hat{\mathbf{H}}_{l s, n}^{\prime}$. The temporal channel estimates are obtained by projecting the pilot channel estimates on to the estimated basis as follows:

$$
\hat{\mathbf{h}}_{n}=\left(\hat{\mathbf{F}}_{n}^{\prime}\right)^{\dagger} \hat{\mathbf{H}}_{l s, n}^{\prime}
$$

where the $(l, k)$ th element of $\hat{\mathbf{F}}_{n}^{\prime}$ is given by

$$
\left[\hat{\mathbf{F}}_{n}^{\prime}\right]_{l, k}=e^{\frac{-j 2 \pi k \hat{\gamma}_{l}}{K T}} \quad \text { for } \quad k \in \mathcal{I}_{i(n)}, \quad l=0,1, \ldots, \hat{L}-1
$$

where $\mathcal{I}_{i(n)}$ is the set of pilot positions (3) chosen for the $n$th OFDM symbol, and $\left\{\hat{\tau}_{l}\right\}_{l=0}^{l=\hat{L}-1}$ are the estimated multipath delays (13). Finally, the frequency-domain channel estimates over all the subcarriers are given by

$$
\hat{\mathbf{H}}_{n}=\hat{\mathbf{F}} \hat{\mathbf{h}}_{n}
$$

where $\hat{\mathbf{h}}_{n}$ is the $\hat{L} \times 1$ estimated channel vector, and the $(l, k)$ th element of $\hat{\mathbf{F}}$ is

$[\hat{\mathbf{F}}]_{l, k}=e^{\frac{-j 2 \pi k \hat{\tau}_{l}}{K T}} \quad$ for $k=0,1, \ldots, K-1, \quad l=0,1, \ldots, \hat{L}-1$.

The channel estimates $\hat{\mathbf{H}}_{n}$ can be further improved by exploiting the time correlation of temporal channel estimates $\hat{\mathbf{h}}_{n}$ using a finite impulse response (FIR) filter. The coefficients of the FIR filter are derived using Weiner filter theory [10].

\section{Simulation AND RESUlts}

The performance of the hopping pilots-based channel estimation scheme is evaluated for an OFDM system having a bandwidth of $B=1 / T=1.75 \mathrm{MHz}, K=128, K_{P}=8(P=16)$, and $L_{\mathrm{cp}}=8$ samples $=4.57 \mu \mathrm{s}$. The OFDM symbol duration is $T_{s}=\left(K+L_{\mathrm{cp}}\right) T=77.7 \mu \mathrm{s}$. Pilot symbols are chosen from quadrature phase-shift keying (QPSK) constellation with power $\Omega=1$. The scalar $\gamma$ is set at 1 . We have considered three types of channel models:

a) $L=5$ with multipath delays uniform over [0, 4.5 $\mu \mathrm{s}]$;

b) $L=3$ with multipath delays uniform over $[0,4.5 \mu \mathrm{s}]$;

c) $L=3$ with multipath delays uniform over $[0,2.3 \mu \mathrm{s}]$.

The first path is always assumed to have a zero delay (i.e., $\tau_{0}=$ 0 ). The multipath power delay profile (pdp) is assumed to be exponential $\operatorname{pdp}(\tau) \sim e^{-\beta \tau}$ with the $\beta=1 / 4$. Each path fades independently according to Jakes' power spectrum [11]. The simulations results presented for two Doppler frequencies of $f_{d}=60 \mathrm{~Hz}$ and $f_{d}=260 \mathrm{~Hz}$. The signal-to-noise ratio (SNR) for the OFDM system is defined based on the total transmit power (by appropriately scaling the channel gain to unity) and the measurement noise variance. The noise variance $\sigma^{2}$ is assumed to be known at the receiver. For every OFDM symbol, the hopping pattern is chosen from the set $\left\{\mathcal{I}_{0}, \mathcal{I}_{1}, \ldots, \mathcal{I}_{P-1}\right\}$ with equal probability. The pilot hopping pattern is assumed to be known at the receiver. A 4-tap FIR filter designed with exact knowledge of Doppler power spectrum is used to exploit time correlation in the channel. (It is found that filter with $>4$ taps the performance improvement in NMSE negligible [10, pp. 535-539].) The performance of the algorithm is evaluated by averaging over 5000 different channel realizations by selecting the multipath delays independently, using the NMSE defined as $\mathrm{NMSE}=\left(E\left[\left\|\mathbf{H}_{n}-\hat{\mathbf{H}}_{n}\right\|^{2}\right] / E\left[\left\|\mathbf{H}_{n}\right\|^{2}\right]\right)$.

Figs. 1 and 2 compare the convergence plots for channel model (a) for fade rates $f_{d}=60 \mathrm{~Hz}$ and $f_{d}=260 \mathrm{~Hz}$, respectively. The pilot hopping-based method is compared with existing method [4] along with DFT-based interpolation [1]. It can be seen that the pilot hopping method converges faster in NMSE compared to the method proposed in [4] for both of the fade rates. The error floor in the DFT-based method is dominated by interpolation errors that cannot be reduced by exploiting time correlation [5, Fig. 7]. We see that the fast convergence of the hopping pilot method is evident in slow fading channels. The fast convergence indicates the fact that the eigenvectors corresponding to the delay subspace are updated faster in the pilot hopping method compared to the fixed pilot method. It is also observed from Fig. 2 that for fast-fading 


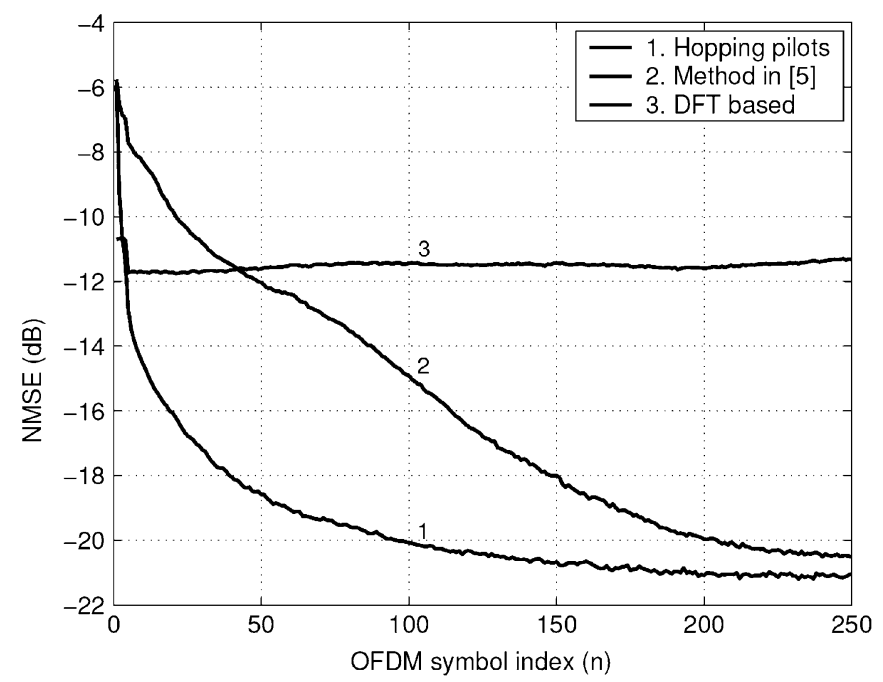

Fig. 1. NMSE convergence plot for channel model (a) at SNR $=15 \mathrm{~dB}$ and $f_{d}=60 \mathrm{~Hz}$.

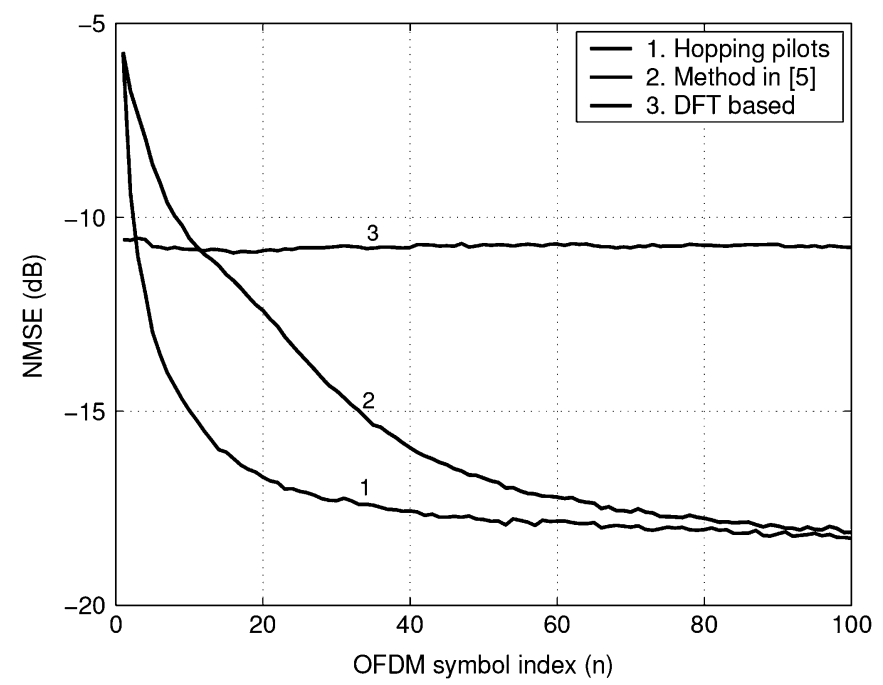

Fig. 2. NMSE convergence plot for channel model (a) at SNR $=15 \mathrm{~dB}$ and $f_{d}=260 \mathrm{~Hz}$.

channels, the curves converge faster but to a higher NMSE compared to Fig. 1. The convergence of the curves to a higher NMSE in Fig. 2 is due to limited time correlation in the channel.

Fig. 3 shows the convergence plots with the pilot hopping technique for channel models (b) and (c). The speedy convergence for the channels with large delay spread can be observed in Fig. 3. Since the phase rotation induced in the path gain due to pilot hopping is proportional to the corresponding delay, the delay subspace is updated at a faster rate for channels with larger delay spread.

\section{CONCLUSION}

In this letter, the effect of hopping pilots on parametric channel estimation in OFDM systems has been investigated. The hopping pilot pattern emulates the effect of channel fading at the receiver and can be exploited for fast multipath delay

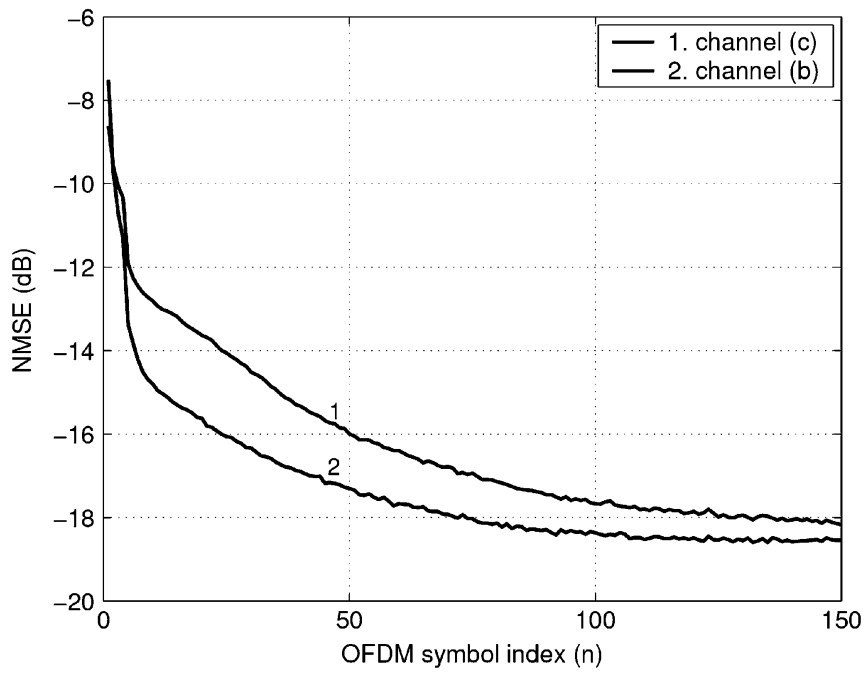

Fig. 3. NMSE convergence plot comparing the effect of channel delay spread on the pilot hopping technique at SNR $=10 \mathrm{~dB}$ and $f_{d}=60 \mathrm{~Hz}$.

estimation. For a channel with $L$ independent paths, $L$ pilot subcarriers are sufficient for channel estimation. The performance results shown that the NMSE of the channel estimates converges faster if the hopping pilot pattern is exploited in the estimation of autocorrelation matrix.

\section{REFERENCES}

[1] J. J. van de Beek, O. Edfors, M. Sandell, S. K. Wilson, and P. O. Borjesson, "On channel estimation in OFDM systems," in Proc. IEEE Vehicular Technology Conf. , vol. 2, Jul. 1995, pp. 815-819.

[2] M. Morelli and U. Mengali, "A comparison of pilot-aided channel estimation methods for OFDM systems," IEEE Trans. Signal Process., vol. 49, no. 12, pp. 3065-3073, Dec. 2001.

[3] M. R. Raghavendra and K. Giridhar, "Improving channel estimation in OFDM systems for sparse multipath channels," IEEE Signal Process. Lett., vol. 12, no. 1, pp. 52-55, Jan. 2005.

[4] B. Yang, K. B. Letaief, R. S. Cheng, and Z. Cao, "Channel estimation for OFDM transmission in multipath fading channels based on parametric channel modeling," IEEE Trans. Commun., vol. 49, no. 3, pp. 467-479, Mar. 2001.

[5] O. Simeone, Y. Bar-Ness, and U. Spagnolini, "Pilot-based channel estimation for OFDM systems by tracking the delay-subspace," IEEE Trans. Wireless Commun., vol. 3, no. 1, pp. 315-325, Jan. 2004.

[6] T. A. Thomas and F. W. Vook, "Broadband MIMO-OFDM channel estimation via near-maximum likelihood time of arrival estimation," in Proc. IEEE Int. Conf. Acoustics, Speech, Signal Processing, vol. 3, May 2002, pp. 2569-2572.

[7] S. Ohno and G. B. Giannakis, "Capacity maximizing MMSE-optimal pilots for wireless OFDM over frequency-selective block Rayleigh-fading channels," IEEE Trans. Inf. Theory, vol. 50, no. 9, pp. 2138-2145, Sep. 2004.

[8] S. U. Pillai and B. H. Kwon, "Forward/backward spatial smoothing techniques for coherent signal identification," IEEE Trans. Acoust., Speech, Signal Process., vol. 37, no. 1, pp. 8-15, Jan. 1989.

[9] R. Roy and T. Kailath, "ESPRIT-Estimation of signal parameters via rotational invariance techniques," IEEE Trans. Acoust., Speech, Signal Process., vol. 37, no. 7, pp. 984-995, Jul. 1989.

[10] L. Hanzo, M. Munster, B. J. Choi, and T. Keller, OFDM and MC-CDMA for Broadband Multi-user Communications, WLAN's and Broadcasting. Piscataway, NJ: IEEE Press/Wiley, 2003.

[11] W. C. Jakes, Microwave Mobile Communications. Piscataway, NJ: IEEE Press, 1993.

[12] U. Reimers, "Digital video broadcasting," IEEE Commun. Mag., vol. 36, no. 6, pp. 104-110, Jun. 1998. 